This item was submitted to Loughborough's Research Repository by the author.

Items in Figshare are protected by copyright, with all rights reserved, unless otherwise indicated.

\title{
Sex differences in postprandial lipaemia after acute high-intensity interval running in young people
}

PLEASE CITE THE PUBLISHED VERSION

https://doi.org/10.1080/02640414.2017.1409610

PUBLISHER

Taylor \& Francis

VERSION

AM (Accepted Manuscript)

\section{PUBLISHER STATEMENT}

This work is made available according to the conditions of the Creative Commons Attribution-NonCommercialNoDerivatives 4.0 International (CC BY-NC-ND 4.0) licence. Full details of this licence are available at: https://creativecommons.org/licenses/by-nc-nd/4.0/

\section{LICENCE}

CC BY-NC-ND 4.0

\section{REPOSITORY RECORD}

Thackray, Alice, Laura Barrett, and Keith Tolfrey. 2019. "Sex Differences in Postprandial Lipaemia After Acute High-intensity Interval Running in Young People”. figshare. https://hdl.handle.net/2134/27300. 


\title{
Sex differences in postprandial lipaemia after acute high-intensity interval running in young people
}

\author{
Alice E. Thackray ${ }^{1}$, Laura A. Barrett ${ }^{1}$ and Keith Tolfrey ${ }^{1}$ \\ ${ }^{1}$ Paediatric Exercise Physiology Research Group, School of Sport, Exercise and Health \\ Sciences, Loughborough University, Loughborough, LE11 3TU, United Kingdom
}

\section{Author details:}

Dr Alice E. Thackray: A.E.Thackray@lboro.ac.uk, 0000-0002-7800-3207

Dr Laura A. Barrett: L.A.Barrett@lboro.ac.uk, 0000-0002-2227-0680

Dr Keith Tolfrey: K.Tolfrey@lboro.ac.uk, 0000-0001-6269-1538

\section{Corresponding author:}

Dr Keith Tolfrey

School of Sport, Exercise and Health Sciences

Loughborough University

Loughborough

\section{LE11 3TU}

United Kingdom

Phone: +44(0)1509 226355

Fax: +44(0)1509 226301

Email: K.Tolfrey@lboro.ac.uk

Running title: Sex differences in postprandial lipaemia

Word count: 4,000 


\title{
Sex differences in postprandial lipaemia after acute high-intensity interval running in young people
}

\begin{abstract}
Acute exercise reduces postprandial triacylglycerol concentrations ([TAG]) in boys and girls; however, it is not known whether between-sex differences exist in response to exercise. Fifteen boys (mean(SD): 11.8(0.4) years) and sixteen girls (12.1(0.7) years) completed two, 2-day conditions. On day 1, participants rested $(\mathrm{CON})$ or completed $10 \times 1$ min high-intensity interval runs at $100 \%$ maximal aerobic speed with 1 min recovery (HIIR). On day 2, participants consumed a standardised breakfast and lunch over a 6.5-h period during which seven capillary blood samples were collected. Based on ratios of the geometric means (95\% CI for ratios), fasting [TAG] was 32\% lower in boys than girls (-44 to $-18 \%$, $\mathrm{ES}=1.31, P<0.001)$, and $12 \%$ lower after HIIR than CON (-18 to $-5 \%$, ES=0.42, $P=0.003$ ); the magnitude of reduction was not significantly different between the sexes ( $8 \%(E S=0.36)$ vs. $15 \%$ ( $E S=0.47)$, respectively; $P=0.29$ ). The total area under the [TAG] versus time curve was $27 \%$ lower in boys than girls ( -40 to $10 \%$, ES $=1.02, P=0.005$ ), and $10 \%$ lower after HIIR than CON (-16 to $-5 \%$, $\mathrm{ES}=0.36, P=0.001$ ); the magnitude of reduction was similar between the sexes (11\% (ES=0.43) vs. $10 \%$ (ES=0.31), respectively; $P=0.87$ ). The small-moderate reduction in postprandial [TAG] after HIIR was similar between the sexes.
\end{abstract}

Keywords: boys; cardiovascular disease risk; girls; triacylglycerol

\section{Introduction}

Elevated postprandial triacylglycerol concentrations ([TAG]) are established as a superior independent risk factor for future cardiovascular disease in adults than traditional fasting concentrations (Bansal et al., 2007). The paediatric origins of atherosclerosis are well established (McGill et al., 2000), and elevated fasting [TAG] in childhood predicts young adult cardiovascular disease (Morrison, Glueck, \& Wang, 2012). Considering the majority of waking hours are spent in a postprandial state, low physical activity and poor diet may exacerbate the risk of future cardiovascular disease with prolonged elevated [TAG]. Therefore, lifestyle interventions that reduce 
postprandial [TAG] and delay precursors of atherosclerotic progression should begin early in life (McGill et al., 2000).

Studies in adults have consistently demonstrated greater postprandial [TAG] in men than women at rest (Couillard et al., 1999; Jackson et al., 2010). Although the mechanisms accounting for this sex difference have not been fully elucidated, greater skeletal muscle uptake and retention of plasma [TAG] (Horton, Commerford, Pagliassotti, \& Bessesen, 2002), lower abdominal visceral adipose tissue accumulation (Couillard et al., 1999), greater suppression of upper-body subcutaneous adipose tissue lipolysis (Jensen, 1995) and/or the protective effect of oestrogen (Westerveld, 1998) in women have been implicated. Nevertheless, Gill, Herd, Tsetsonis, and Hardman (2002) pooled data and compared results from 38 men and 43 women and reported that the exercise-induced reduction in postprandial [TAG] was not different between men and women (23.5\% vs. $19.8 \%$, respectively).

Acute moderate- to high-intensity exercise performed the day before a standardised meal reduces postprandial [TAG] in boys and girls (Tolfrey, Thackray, \& Barrett, 2014b), highlighting the potential for exercise to promote a healthier cardiovascular risk profile at a time when many adverse health behaviours can become established. However, it is not known whether the postprandial TAG response is different in boys and girls the day following a single bout of exercise. This represents an important gap in our understanding of this marker of future cardiovascular disease risk in young people, and may have important implications in terms of identifying underlying sex differences in the physiological response to exercise during this critical period of growth and development. 
Therefore, the aim of this study was to compare directly the postprandial TAG response in healthy 11- to 13-year-old boys and girls, and to examine the magnitude of change in postprandial [TAG] following a single bout of exercise.

\section{Methods}

\section{Participants}

After approval from the University Ethical Advisory Committee, 15 boys (11.3 to 12.9 years) and 16 girls (11.3 to 13.3 years) volunteered to participate in this study conducted at Loughborough University, United Kingdom. Written assent from participants and written informed consent from a parent or guardian was obtained before the study commenced. All participants were in good health and were generally physically active, but not specifically accustomed to high-intensity running. Physical and physiological characteristics are presented in Table 1.

\section{Anthropometry and physical maturation}

Stature was measured to the nearest $0.01 \mathrm{~m}$ using a fixed stadiometer (Holtain Ltd, Crosswell, UK), body mass was quantified to the nearest $0.1 \mathrm{~kg}$ using a digital scale (Seca 770, Seca Ltd, Hamburg, Germany) and body mass index was calculated as body mass (kg) divided by stature (m) squared. Skinfold thickness was measured at the triceps and subscapular to the nearest $0.2 \mathrm{~mm}$ using Harpenden callipers (Baty International, West Sussex, UK). All measurements were taken on the right-hand side of the body, and the median of three measurements at each site was used to estimate percent body fat (Slaughter et al., 1988).

Participants provided a self-assessment of their level of physical maturity using drawings depicting the five stages of pubic hair development, ranging from 1 indicating 
pre-pubescence to 5 indicating full sexual maturity (Tanner, 1962). The median (interquartile range) stage of pubic hair development was 2(1) for boys (stage1: $n=2$; stage 2: $n=11$; stage 3: $n=2$ ) and 2(3) for girls (stage 1: $n=4$; stage 2: $n=6$; stage 3: $n=1$; stage 4: $n=3$; stage 5: $n=2$ ). Menstrual cycle phase was not controlled in the female participants because 12 of the 16 girls were premenarcheal, and two of the remaining four girls reported that their menstrual cycle was sporadic and irregular.

\section{Preliminary exercise measurements}

Participants were familiarised with walking and running on the treadmill (h/p/cosmos mercury med, Nussdorf-Traunstein, Germany) prior to completing an incremental speed-based protocol to determine peak oxygen uptake ( $\left.\dot{\mathrm{V}}_{2}\right)$ and maximal aerobic speed (MAS). The protocol started at $5.0 \mathrm{~km} \cdot \mathrm{h}^{-1}$ for girls and $6.0 \mathrm{~km} \cdot \mathrm{h}^{-1}$ for boys with the treadmill gradient set at $1 \%$ throughout and the treadmill speed was increased in 0.5 $\mathrm{km} \cdot \mathrm{h}^{-1}$ increments every $30 \mathrm{~s}$ until volitional exhaustion. Heart rate was recorded using short-range telemetry (Polar PE 4000, Kempele, Finland), ratings of perceived exertion (RPE) were recorded in the last 10 s of each 30 s stage using Borg's 6-20 scale, and expired air samples were monitored continuously using an online breath-by-breath gas analysis system (Metalyzer 3B, Cortex, Leipzig, Germany). An average of the breathby-breath $\dot{\mathrm{V}} \mathrm{O}_{2}$ data was taken every $10 \mathrm{~s}$, and peak $\dot{\mathrm{V}} \mathrm{O}_{2}$ was defined as the highest $30 \mathrm{~s}$ rolling average; the treadmill speed corresponding to peak $\dot{\mathrm{VO}}_{2}$ was recorded as MAS.

\section{Experimental design}

Using a within measures, crossover design, participants completed two, 2-day experimental conditions separated by at least 14 days: rest control (CON) and highintensity interval running (HIIR). The study design is presented in Figure 1. 


\section{Day 1: intervention day}

Participants reported to the laboratory at 15:30 and completed all measures by 17:30.

Body mass was quantified upon arrival to standardise the meals provided on day 2 (described below). During CON, participants rested for the duration of the visit. During HIIR, a 5 min warm-up was completed at 60\% MAS followed immediately by the acute high-intensity running intervals. The high-intensity running comprised $10 \times 1 \mathrm{~min}$ treadmill runs at 100\% MAS, with 1 min active recovery between intervals. Participants dismounted the treadmill during the recovery periods and were encouraged to pace around the lab to avoid venous pooling and feeling light-headed. Heart rate was monitored continuously and RPE was recorded in the last $10 \mathrm{~s}$ of each running interval as described previously.

\section{Standardisation of dietary intake and physical activity}

Participants weighed, recorded and replicated their habitual dietary intake during the 48-h period (pre-intervention and intervention day) before day 2 of both experimental conditions. Two-day diet records were analysed using dietary analysis software (CompEat Pro Version 5.8.0, Nutrition Systems, Banbury, UK).

Participants consumed a cereal snack bar (1.2 g fat, 15.9 g carbohydrate, $1.0 \mathrm{~g}$ protein, $335 \mathrm{~kJ}$ energy) at 19:45 on the intervention day to standardise the overnight fasting period. Participants were allowed to drink plain water, but no other drinks or food, before arriving at the laboratory on day 2 .

Participants recorded all physical activity categorised according to intensity level during the pre-intervention and intervention day of the first condition. Participants were asked to minimise their physical activity during this period, and the activity pattern was replicated before the subsequent condition. 


\section{Day 2: postprandial day}

After a 12-h overnight fast, participants arrived at the laboratory at 07:45 and provided a fasting capillary blood sample after 10 min seated rest. A standardised breakfast was consumed within 15 min marking the start of the postprandial period (08:00) (Figure 1). Breakfast consisted of croissants, chocolate spread, whole milk, double cream and milkshake powder. The meal quantity was prescribed relative to body mass and provided $1.5 \mathrm{~g}$ fat (60.7\% of meal total energy), $1.8 \mathrm{~g}$ carbohydrate (32.6\%), $0.4 \mathrm{~g}$ protein (6.7\%) and $93 \mathrm{~kJ}$ energy per kilogram body mass. Subsequent capillary blood samples were taken at $0.5,1,3,4.5,5$ and 6.5 -h following the start of the breakfast, and participants consumed a standardised lunch, within 20 min, at 4-h (Figure 1). Lunch consisted of white bread, butter, cheese, potato crisps, whole milk and milkshake powder, and provided $1.2 \mathrm{~g}$ fat (51.9\%), $1.9 \mathrm{~g}$ carbohydrate (36.5\%), $0.6 \mathrm{~g}$ protein (11.6\%) and $89 \mathrm{~kJ}$ energy per kilogram body mass. Participants rested throughout the day and were able to read, watch DVD films and play non-active computer games. Participants consumed water ad libitum in the postprandial period of the first condition; the ingested volume was replicated in the subsequent condition.

\section{Analytical methods}

After the hand was pre-warmed for $5 \mathrm{~min}$ in water heated to $40^{\circ} \mathrm{C}$, the fingertip was pierced (Unistick 3 Extra, Owen Mumford, Oxford, UK) and $600 \mu \mathrm{L}$ whole capillary blood was collected into potassium EDTA coated Microvette CB300 tubes (Sarstedt Ltd, Leicester, UK). The whole blood samples were immediately centrifuged at $12,800 \mathrm{~g}$ for 15 min (Eppendorf 5415c, Hamburg, Germany) and the resulting plasma was stored at $-80^{\circ} \mathrm{C}$ for up to two months before subsequent analyses. Plasma [TAG] and glucose concentrations ([glucose]) were analysed by enzymatic, colorimetric methods using a 
benchtop analyser (Pentra 400, HORIBA ABX Diagnostics, Montpellier, France). The within-batch coefficient of variation for plasma [TAG] and [glucose] were 1.0 and $0.4 \%$, respectively for boys and 1.6 and $0.8 \%$, respectively for girls. Haemoglobin concentration and haematocrit were quantified in duplicate at 0 and 6.5-h to estimate the acute change in plasma volume (Dill \& Costill, 1974).

\section{Statistical analyses}

Data were analysed using SPSS Statistics 21.0 (IBM Corporation, New York, USA). The trapezium rule was used to calculate the total area under the curve for TAG (TAUC-TAG) and glucose (TAUC-glucose). The incremental area under the curve (iAUC) was calculated using the same method after adjusting for fasting concentrations. Normality of the data was checked using Shapiro Wilk tests. Normally distributed data are presented as mean (SD). Concentrations of TAG and glucose were not normally distributed and were natural log transformed prior to analysis. These data are presented as geometric mean (95\% confidence intervals (CI)) and analysis is based on ratios of the geometric means and 95\% CI for ratios.

Physical and physiological characteristics and HIIR responses were compared between groups using linear mixed models with sex as the sole factor. Physical maturity was compared between groups using a non-parametric Mann Whitney-U test and presented as median (interquartile range). The 95\% CI was derived using a bootstrapping method (Table 1).

Energy and macronutrient intakes, estimated changes in plasma volume and fasting concentrations were compared using linear mixed models with fixed factors for sex and condition. Differences in postprandial plasma [TAG] and [glucose] were examined using three separate approaches: 1 ) differences over the $6.5 \mathrm{~h}$ postprandial 
period ( $2 \times 2 \times 7$ condition-by-sex-by-time linear mixed model); 2$)$ TAUC $(2 \times 2$ condition-by-sex linear mixed model); and 3) iAUC $(2 \times 2$ condition-by-sex linear mixed model). Where significant main effects of condition or sex were identified for the TAUC or iAUC analysis, temporal changes were examined over sub-sections of the total postprandial period (0-1 h, 1-4.5 h, 4.5-6.5 h) using separate linear mixed models with sex and condition included as fixed factors. All linear mixed models were adjusted appropriately for the period effect (Senn, 1993).

Bivariate correlations identifying possible determinants of the exercise-induced changes in TAUC-TAG were quantified using Pearson’s product moment correlations. Statistical significance was accepted as $P<0.05$. Absolute standardised effect sizes (ES) are included to supplement important findings, with an ES of 0.2 considered the minimum important difference, 0.5 moderate and 0.8 large (Cohen, 1988).

\section{Results}

\section{Participant characteristics}

Girls were marginally older than boys (ES=0.49, $P=0.19$ ), and percent body fat was lower in boys compared with girls $(\mathrm{ES}=1.75, P<0.001)$ (Table 1). Peak $\dot{\mathrm{VO}} 2$ was higher, on average, in boys than girls (ES=2.08, $P<0.001$ ), with a trend for MAS to be higher in boys than girls (ES=0.70, $P=0.06$ ) (Table 1$)$. The other measured physical and physiological variables were similar between the sexes $(P \geq 0.41)$ (Table 1$)$.

\section{Dietary intake}

Energy and macronutrient intakes were similar on the pre-intervention day across conditions and groups (main effect condition $P \geq 0.11$; main effect sex $P \geq 0.08$; condition-by-sex interaction $P \geq 0.36$ ). Energy and macronutrient intakes during the 
intervention day are displayed in Table 2. Energy and macronutrient intakes were similar between conditions ( $P \geq 0.69$ ). Energy intake ( $\mathrm{ES}=1.02, P=0.003)$, and absolute intakes of protein (ES=0.83, $P=0.03)$, carbohydrate $(\mathrm{ES}=0.67, P=0.06)$ and fat ( $\mathrm{ES}=0.97, P=0.004)$ were greater in boys than girls. The relative percentage contribution of protein, carbohydrate and fat to total energy intake was similar between conditions ( $P \geq 0.23)$; however, the relative contributions of carbohydrate and fat to total energy intake were lower ( $\mathrm{ES}=0.91, P=0.02$ ) and higher ( $\mathrm{ES}=0.72, P=0.05$ ), respectively, in boys than girls.

\section{Exercise responses}

Exercise responses are displayed in Table 3. Peak heart rate determined during the speed-based incremental treadmill protocol was meaningfully, albeit not significantly, lower in boys than girls (ES=0.57, $P=0.13$ ). During HIIR, average start interval heart rate was lower in boys than girls when expressed in beats $\mathrm{min}^{-1}(\mathrm{ES}=1.73, P<0.001$ ), and as a percentage of peak heart rate $(\mathrm{ES}=1.65, P<0.001)$. Average end interval heart rate was lower in boys than girls (ES=0.75, $P=0.05$ ), but was not significantly different when expressed as a percentage of peak heart rate $(P=0.25)$. Average RPE over the 10 intervals was greater in boys than girls ( $\mathrm{ES}=0.85, P=0.03$ ).

\section{Fasting plasma concentrations}

Average changes in plasma volume between the fasting and 6.5-h postprandial samples were not different across conditions or groups (main effect condition $P=0.88$; main effect sex $P=0.44$; condition-by-sex interaction $P=0.95$ ). Therefore, the raw plasma concentrations were used in all statistical analyses without adjustment. Fasting [TAG] and [glucose] are displayed in Table 4. Fasting [TAG] was 32\% lower in boys than girls 
(main effect sex: $\mathrm{ES}=1.31, P<0.001$ ) and 12\% lower after HIIR than CON (main effect condition: $\mathrm{ES}=0.42, P=0.003)$. Despite a seemingly greater reduction in fasting [TAG] in the girls (15\%, ES=0.47) than boys (8\%, ES=0.36), this difference was not statistically significant (condition-by-sex interaction $P=0.29$ ). Fasting [glucose] was similar across conditions and groups (main effect condition $P=0.33$; main effect sex $P=0.13$; condition-by-sex interaction $P=0.66$ ).

\section{Postprandial plasma concentrations}

Linear mixed models for postprandial plasma [TAG] over time revealed significant main effects of condition $(P<0.001)$, sex $(P=0.004)$ and time $(P<0.001)$, but not a condition-by-sex interaction ( $P=0.60$ ) (Figure 2). The TAUC-TAG was $27 \%$ lower in boys than girls (main effect sex: ES=1.02, $P=0.005$ ) and 10\% lower after HIIR than CON (main effect condition: $E S=0.36, P=0.001$ ), but the magnitude of reduction following HIIR was similar in boys and girls (11\% (ES=0.43) vs. 10\% (ES=0.31), respectively; condition-by-sex interaction $P=0.87$ ) (Table 4). The TAUC-TAG was lower in boys than girls between $0-1$ h by $26 \%$ (-39 to $-10 \%$, ES=1.03, $P=0.004), 1-4.5$ h by $32 \%$ ( -45 to $-14 \%$, ES=1.16, $P=0.002)$ and $4.5-6.5$ h by $18 \%$ ( -33 to $-1 \%$, ES=0.68, $P=0.04)$. The TAUC-TAG was lower in HIIR than CON between $0-1 \mathrm{~h}$ by $11 \%$ (-16 to $-5 \%$, ES=0.40, $P=0.001), 1-4.5 \mathrm{~h}$ by $11 \%(-17$ to $-5 \%, \mathrm{ES}=0.35, P=0.001)$ and $4.5-6.5 \mathrm{~h}$ by $9 \%(-15$ to $-2 \%, E S=0.32, P=0.01)$. The iAUC-TAG was similar across conditions and groups (main effect condition $P=0.73$; main effect sex $P=0.78$; condition-by-sex interaction $P=0.19$ ) (Table 4).

Linear mixed models for plasma [glucose] revealed no main effects of condition or sex when analysed over the $6.5 \mathrm{~h}$ postprandial period or summarized in the TAUCglucose (Table 4) (main effect condition $P \geq 0.19$; main effect sex $P \geq 0.31$ ). However, the 
condition-by-sex interactions were significant for plasma [glucose] over the $6.5 \mathrm{~h}$ postprandial period ( $P=0.04)$ and near significant for TAUC-glucose $(P=0.06)$, with $-1 \%$ vs. $3 \%$ changes for both outcome variables in boys and girls, respectively. The iAUC-glucose was similar across conditions and groups (main effect condition $P=0.27$; main effect sex $P=0.28$; condition-by-sex interaction $P=0.23$ ) (Table 4).

\section{Individual changes and predictors of the HIIR-induced change in TAUC-TAG}

Individual changes (delta) in TAUC-TAG between HIIR and CON for boys and girls are shown in Figure 3. The reduction in TAUC-TAG following HIIR was greater than changes in CON for ten (67\%) boys and ten (63\%) girls. End interval percent peak heart rate demonstrated an inverse relationship with delta TAUC-TAG $(\mathrm{r}=-0.44, P=0.01)$. The HIIR-induced change in fasting [TAG] was positively correlated with the change in TAUC-TAG relative to CON ( $\mathrm{r}=0.50, P=0.005)$.

\section{Discussion}

The primary finding of the present study was that the magnitude of reduction in postprandial [TAG] after a single session of HIIR was similar in 11- to 13-year-old boys and girls $(11 \%(\mathrm{ES}=0.43)$ vs. $10 \%$ ( $\mathrm{ES}=0.31)$, respectively). The small-to-moderate reductions in fasting [TAG] after HIIR were not significantly different between the sexes (8\% (ES=0.36) vs. 15\% (ES=0.47) in boys and girls, respectively). However, the main effect of sex revealed that fasting and postprandial [TAG] were lower in boys than girls.

The HIIR-induced reduction in fasting [TAG] supports the majority of previous exercise postprandial studies in young people (Lee, Burns, White, Kuk, \& Arslanian, 2013; Tolfrey et al., 2014a). The higher fasting [TAG] in girls compared with boys, whilst also evident elsewhere in the literature (Boddy et al., 2014), may have 
contributed to the heightened postprandial TAG response (Couch et al., 2000). However, substantial intra-individual variation is evident in childhood fasting [TAG] (Tolfrey, Campbell, \& Jones, 1999), and impaired postprandial TAG clearance is associated independently with an increased risk of cardiovascular disease (Bansal et al., 2007). Therefore, postprandial [TAG] may be more informative of metabolic health in young people.

The lower postprandial [TAG] in boys than girls contrasts previous findings in adults, which have demonstrated consistently that premenopausal women experience lower postprandial [TAG] than men (Couillard et al., 1999; Jackson et al., 2010). Recent studies in adolescents are less consistent, with one study reporting a lower TAUC-TAG response in girls than boys (Bond et al., 2015c), whereas another study reported no difference (Bond et al., 2015b). The reason for the contrasting findings in young people is unclear, but the slightly older age group ( 14 years) recruited in the studies by Bond et al. (2015b, 2015c) may influence the postprandial lipaemic response. Nevertheless, the time-zone analysis in this study revealed lower postprandial [TAG] in the boys than girls throughout the entire 6.5-h postprandial period.

While the mechanistic pathways underlying the lower postprandial [TAG] in boys cannot be determined directly from this study, differences in biological maturation, body composition, cardiorespiratory fitness and/or dietary intake may be implicated. A transient state of insulin resistance occurs during puberty in both sexes, which typically peaks at pubertal stage 3 and girls appear more insulin resistant than boys at all stages of sexual maturation (Moran et al., 1999). Considering insulin resistance is an independent predictor of postprandial [TAG] in healthy adults (Jeppesen et al., 1995), it is possible that greater insulin resistance in girls may amplify the postprandial lipaemic response compared with similarly aged boys. Although not significantly different, a 
wider range of self-assessed biological maturity ratings were identified in the girls. Girls enter and end puberty approximately two years before boys and pubertal events do not occur in the same sequence between the sexes (Baxter-Jones, Eisenmann, \& Sherar, 2005). Consequently, it is difficult to align girls and boys for pubertal status when making between-sex comparisons (Baxter-Jones et al., 2005). Whilst the limitations of self-assessed sexual maturation may have restricted the ability to detect subtle betweengroup differences in this study, previous evidence suggests this method provides an accurate and reliable surrogate measure of biological maturation (Matsudo \& Matsudo, 1994). A priori matching of the young participants based on biological maturation would be the best way to address this study limitation, but this would be very difficult logistically and ethically too. Nevertheless, a positive association between sexual maturation and fasting [TAG] in 10- to 13-year-old boys and girls has been reported previously (Bertrais et al., 2000); therefore, it seems plausible that biological maturation may influence postprandial lipaemia in young people. Previous research has also demonstrated that menstrual cycle phase exerts a meaningful influence on postprandial lipaemia in women (Gill, Malkova, \& Hardman, 2005), although there is conflicting evidence (Wendler, Michel, Kästner, \& Schmahl, 1992). The majority of the girls in this study were premenarcheal and, therefore, the potential confounding effects of cyclical hormone changes on the between condition and sex comparisons were minimised.

In accordance with previous studies in this age group (Bond et al., 2015b, 2015c; Kelly et al., 2014), percent body fat was significantly lower in boys compared with girls. Although the values for boys and girls were within the optimal range for health (McCarthy, Cole, Fry, Jebb, \& Prentice, 2006), percent body fat has been identified as a strong determinant of insulin resistance in children (Arslanian \& Suprasongsin, 1996). Furthermore, measures of adiposity are associated with 
cardiovascular disease risk factors, including fasting [TAG], in young people (Steinberger et al., 2005). The reported between-sex differences in body fat distribution may be more enlightening in explaining the sex dimorphism in postprandial [TAG] seen in the present study (Huang, Johnson, Figueroa-Colon, Dwyer, \& Goran, 2001). In this regard, visceral adipose tissue has been identified as the strongest predictor of postprandial [TAG] in overweight white adolescents, but the limited sample size precluded a direct sex comparison (Lee et al., 2013).

Alternatively, it is possible that the greater peak $\dot{\mathrm{V}}{ }_{2}$ in boys (Table 1) confers a protective effect on postprandial lipaemia; however, cardiorespiratory fitness has not been identified as a predictor of postprandial [TAG] in adolescents (Bond et al., 2015b, 2015c) or adults (Gill et al., 2002). Finally, although dietary intake was different on the intervention day between the sexes (Table 2), energy and macronutrient intakes were all greater in boys than girls, supporting previous evidence in similarly aged young people (Greatwood, Daly-Smith, McGregor, \& McKenna, 2013), and the relative proportion of carbohydrate, a potent stimulator of [TAG], was lower in boys. Therefore, this component is unlikely to be an influencing factor based on the available evidence. While a number of potential explanations have been highlighted, further work is required to elucidate the precise mechanisms explaining the exaggerated postprandial TAG profile in girls.

The reduction in postprandial [TAG] following HIIR supports several studies in adults adopting acute intermittent, high-intensity exercise protocols; however, this finding is not universal (reviewed by Burns, Miyashita, \& Stensel, 2015). Recent evidence in 12- to 14-year-old boys reported a reduction in postprandial [TAG] after a single session of repeated maximal cycle sprints (Sedgwick, Morris, Nevill, \& Barrett, 2015). In contrast to our findings and those of Sedgwick et al. (2015), Bond et al. 
(2015b, 2015c) reported no effect of moderate- or high-intensity exercise on TAUCTAG in boys and girls, although iAUC-TAG was significantly lower following exercise in girls, but not boys (Bond et al., 2015b). This finding is surprising considering the majority of studies support the TAG-lowering effect of acute moderate- to vigorousintensity exercise in boys and girls (Tolfrey et al., 2014). However, this disparity may be explained by the timing of the exercise session completed $\sim 1$-h before the high-fat meal (Bond et al., 2015b) or accumulated during the postprandial period (Bond et al., 2015c). A further study in adolescent boys and girls isolated the acute effect of a twoweek high-intensity intermittent cycling intervention from the chronic training adaptation by including a postprandial assessment the day after the last exercise bout (Bond et al. 2015a). Although the postprandial TAG profile the day after exercise was not significantly different to the pre-intervention response (Bond et al. 2015a), the marked difference in study design limits the ability to make direct comparisons with the acute-based literature. Our findings extend the evidence base by demonstrating that the acute HIIR-induced reduction in postprandial [TAG] was similar in boys and girls (11\% vs. 10\%, respectively; Table 4, Figure 2), and supports the similar moderate-intensity exercise-induced reduction in postprandial [TAG] reported in men and women (23.5\% vs. 19.8\%, respectively) (Gill et al., 2002). Therefore, the findings of this study suggest that 11- to 13-year-old boys and girls may acquire similar metabolic health benefits in postprandial TAG metabolism the day following acute HIIR.

The mechanisms responsible for the HIIR-induced reduction in postprandial [TAG] were not measured directly in this study due to the invasive nature of the techniques required. In adults, increased clearance of circulating TAG facilitated by enhanced lipoprotein lipase (LPL) activity and/or the secretion of fewer, TAG-richer very low-density lipoproteins (VLDL) have been implicated (Maraki \& Sidossis, 2013). 
Recent evidence indicates that exercise appears to increase the affinity of large VLDL for LPL-mediated hydrolysis in the fasted and postprandial state, which is likely to contribute to the exercise-induced reduction in [TAG] (Ghafouri et al., 2015). Indeed, the meaningful relationship between the HIIR-induced change in fasting [TAG] and TAUC-TAG ( $\mathrm{r}=0.50, P=0.005$ ), and the small differences in iAUC-TAG between conditions may point to changes in endogenous and not exogenous TAG pathways (Davitt, Arent, Tuazon, Golem, \& Henderson, 2013).

Although the clinical significance of our findings cannot be elucidated, the majority of the postprandial [TAG] (97\%) were below the $2.3 \mathrm{mmol} \cdot \mathrm{L}^{-1}$ threshold proposed as a desirable concentration in young people (Kolovou, Bilianou, \& Mikhailidis, 2011). Postprandial [TAG] are established as an independent risk marker for future cardiovascular disease (Bansal et al., 2007), and the majority of young people do not meet the current physical activity guidelines (Hallal et al., 2012). Therefore, the potential for a single session of HIIR to reduce an important marker of future atherogenic risk in boys and girls is encouraging.

The inter-individual variability in postprandial [TAG] following HIIR (Figure 3) supports the heterogeneous response reported in previous exercise postprandial studies in young people (Tolfrey et al., 2014a) and adults (Gill et al., 2002). A similar proportion of boys and girls experienced a HIIR-induced reduction in postprandial [TAG] ( 65\%; Figure 3). Average end-interval percent peak heart rate was significantly correlated with delta TAUC-TAG suggesting that exercising at a higher relative intensity augments the reduction in postprandial [TAG]. Consequently, small increases in effort could afford greater potential gains in long-term health providing the exercise stimulus can be applied regularly without risking injury or burn-out in the young participants. 


\section{Conclusions}

Fasting and postprandial [TAG] were lower in healthy 11- to 12-year-old boys compared with similarly aged girls, but the small-to-moderate reduction following acute HIIR was not significantly or meaningfully different between the sexes. This suggests that boys and girls can experience similar metabolic health benefits in fasting and postprandial TAG metabolism the day after a single bout of HIIR.

\section{Acknowledgements}

We thank Woodbrook Vale High School and Rawlins Academy in Loughborough for their support and understanding throughout this research. We also thank the participants and their parents for their commitment throughout this research.

\section{Funding details}

The research was supported by the National Institute for Health Research (NIHR) Leicester Biomedical Research Centre. The views expressed are those of the authors and not necessarily those of the NHS, the NIHR or the Department of Health.

\section{Disclosure of interest}

The authors report no conflicts of interest.

\section{References}

Arslanian, S., \& Suprasongsin, C. (1996). Insulin sensitivity, lipids, and body composition in childhood: is "syndrome X" present? The Journal of Clinical Endocrinology and Metabolism, 81, 1058-1062. doi:10.1210/jcem.81.3.8772576

Bansal, S., Buring, J.E., Rifai, N., Mora, S., Sacks, F.M., \& Ridker, P.M. (2007). Fasting compared with nonfasting triglycerides and risk of cardiovascular events in 
women. The Journal of the American Medical Association, 298, 309-316.

doi:10.1001/jama.298.3.309

Baxter-Jones, A.D.G., Eisenmann, J.C., \& Sherar, L.B. (2005). Controlling for maturation in pediatric exercise science. Pediatric Exercise Science, 17, 18-30. doi:10.1123/pes.17.1.18

Bertrais, S., Balkau, B., Charles, M.A., Vol, S., Calvet, C., Tichet, J., \& Eschwege, E. (2000). Puberty-associated differences in total cholesterol and triglyceride levels according to sex in French children aged 10-13 years. Annals of Epidemiology, 10, $316-323$.

Boddy, L.M., Murphy, M.H., Cunningham, C., Breslin, G., Foweather, L., Gobbi, R., ... Stratton, G. (2014). Physical activity, cardiorespiratory fitness, and clustered cardiometabolic risk in 10- to 12-year-old school children: the REACH Y6 study. American Journal of Human Biology, 26, 446-451. doi:10.1002/ajhb.22537

Bond, B., Cockcroft, E.J., Williams, C.A., Harris, S., Gates, P.E., Jackman, S.R., ... Barker, A.R. (2015a). Two weeks of high-intensity interval training improves novel but not traditional cardiovascular disease risk factors in adolescents. American Journal of Physiology. Heart and Circulatory Physiology, 309, H1039-H1047. doi:10.1152/ajpheart.00360.2015

Bond, B., Williams, C.A., Isic, C., Jackman, S.R., Tolfrey, K., Barrett, L.A., \& Barker, A.R. (2015b). Exercise intensity and postprandial health outcomes in adolescents. European Journal of Applied Physiology, 115, 927-936. doi:10.1007/s00421-014-3074$\underline{8}$ 
Bond, B., Williams, C.A., Jackman, S.R., Woodward, A., Armstrong, N., \& Barker, A.R. (2015c). Accumulating exercise and postprandial health in adolescents.

Metabolism, 64, 1068-1076. doi:10.1016/j.metabol.2015.05.016

Burns, S.F., Miyashita, M., \& Stensel, D.J. (2015). High-intensity interval exercise and postprandial triacylglycerol. Sports Medicine, 45, 957-968. doi:10.1007/s40279-015$\underline{0327-6}$

Cohen, J. (1988). Statistical power analysis for the behavioural sciences (2nd ed.). Hillsdale, New Jersey: Lawrence Erlbaum Associates.

Couch, S.C., Isasi, C.R., Karmally, W., Blaner, W.S., Starc, T.J., Kaluski, D., ... Berglund, L. (2000). Predictors of postprandial triacylglycerol response in children: the Columbia University Biomarkers Study. The American Journal of Clinical Nutrition, 72, 1119-1127.

Couillard, C., Bergeron, N., Prud’homme, D., Bergeron, J., Tremblay, A., Bouchard, C., ... Després, J.P. (1999). Gender difference in postprandial lipemia: importance of visceral adipose tissue accumulation. Arteriosclerosis, Thrombosis, and Vascular Biology, 19, 2448-2455. doi:10.1161/01.ATV.19.10.2448

Davitt, P.M., Arent, S.M., Tuazon, M.A., Golem, D.L., \& Henderson, G.C. (2013). Postprandial triglyceride and free fatty acid metabolism in obese women after either endurance or resistance exercise. Journal of Applied Physiology, 114, 1743-1754. doi:10.1152/japplphysiol.00095.2013

Dill, D.B., \& Costill, D.L. (1974). Calculation of percentage changes in volumes of blood, plasma, and red cells in dehydration. Journal of Applied Physiology, 37, 247248. 
Ghafouri, K., Cooney, J., Bedford, D.K., Wilson, J., Caslake, M.J., \& Gill, J.M.R. (2015). Moderate exercise increases affinity of large very low-density lipoproteins for hydrolysis by lipoprotein lipase. The Journal of Clinical Endocrinology and Metabolism, 100, 2205-2213. doi:10.1210/jc.2015-1196

Gill, J.M.R., Herd, S.L., Tsetsonis, N.V., \& Hardman, A.E. (2002). Are the reductions in triacylglycerol and insulin levels after exercise related? Clinical Science (London), 102, 223-231. doi: $\underline{10.1042 / \operatorname{cs} 1020223}$

Gill, J.M.R., Malkova, D., \& Hardman, A.E. (2005). Reproducibility of an oral fat tolerance test is influenced by phase of menstrual cycle. Hormone and Metabolic Research, 37, 336-341. doi:10.1055/s-2005-861481

Greatwood, H.C., Daly-Smith, A., McGregor, S., \& McKenna, J. (2013). Year 7 dietary intake: a comparison of two schools with middle-high socio-economic status. Journal of Human Nutrition and Dietetics, 26, 563-569. doi:10.1111/jhn.12124

Hallal, P.C., Andersen, L.B., Bull, F.C., Guthold, R., Haskell, W., \& Ekelund, U. (2012). Global physical activity levels: surveillance progress, pitfalls, and prospects. Lancet, 380, 247-257. doi:10.1016/S0140-6736(12)60646-1

Horton, T.J., Commerford, S.R., Pagliassotti, M.J., \& Bessesen, D.H. (2002). Postprandial leg uptake of triglyceride is greater in women than in men. American Journal of Physiology. Endocrinology and Metabolism, 283, E1192-E1202. doi:10.1152/ajpendo.00164.2002

Huang, T.T.K., Johnson, M.S., Figueroa-Colon, R., Dwyer, J.H., \& Goran, M.I. (2001). Growth of visceral fat, subcutaneous abdominal fat, and total body fat in children. Obesity Research, 9, 283-289. doi:10.1038/oby.2001.35 
Jackson, K.G., Clarke, D.T., Murray, P., Lovegrove, J.A., O’Malley, B., Minihane, A.M., \& Williams, C.M. (2010). Introduction to the DISRUPT postprandial database: subjects, studies and methodologies. Genes and Nutrition, 5, 39-48.

doi:10.1007/s12263-009-0149-y

Jensen, M.D. (1995). Gender differences in regional fatty acid metabolism before and after meal ingestion. The Journal of Clinical Investigation, 96, 2297-2303.

doi:10.1172/JCI118285

Jeppesen, J., Hollenbeck, C.B., Zhou, M.Y., Coulston, A.M., Jones, C., Chen, Y.D., \& Reaven, G.M. (1995). Relation between insulin resistance, hyperinsulinemia, postheparin plasma lipoprotein lipase activity, and postprandial lipemia. Arteriosclerosis, Thrombosis, and Vascular Biology, 15, 320-324. doi:10.1161/01.ATV.15.3.320

Kelly, A.S., Dengel, D.R., Hodges, J., Zhang, L., Moran, A., Chow, L., ... Steinberger, J. (2014). The relative contributions of the abdominal visceral and subcutaneous fat depots to cardiometabolic risk in youth. Clinical Obesity, 4, 101-107. doi: $10.1111 /$ cob.12044

Kolovou, G.D., Bilianou, H., \& Mikhailidis, D.P. (2011). Postprandial lipemia in children and adolescents. Current Vascular Pharmacology, 9, 318-320. doi: $\underline{10.2174 / 157016111795495521}$

Lee, S., Burns, S.F., White, D., Kuk, J.L., \& Arslanian, S. (2013). Effects of acute exercise on postprandial triglyceride response after a high-fat meal in overweight black and white adolescents. International Journal of Obesity, 37, 966-971.

doi:10.1038/ijo.2013.29 
Maraki, M.I., \& Sidossis, L.S. (2013). The latest on the effect of prior exercise on postprandial lipaemia. Sports Medicine, 43, 463-481. doi:10.1007/s40279-013-0046-9

Matsudo, S.M.M., \& Matsudo, V.K.R. (1994). Self-assessment and physician assessment of sexual maturation in Brazilian boys and girls: concordance and reproducibility. American Journal of Human Biology, 6, 451-455. doi:10.1002/ajhb.1310060406

McCarthy, H.D., Cole, T.J., Fry, T., Jebb, S.A., \& Prentice, A.M. (2006). Body fat reference curves for children. International Journal of Obesity, 30, 598-602. doi:10.1038/sj.ijo.0803232

McGill, H.C., McMahan, C.A., Herderick, E.E., Malcom, G.T., Tracy, R.E., \& Strong, J.P. (2000). Origin of atherosclerosis in childhood and adolescence. The American Journal of Clinical Nutrition, 72, 1307S-1315S.

Moran, A., Jacobs, D.R., Steinberger, J., Hong, C.P., Prineas, R., Luepker, R., \& Sinaiko, A.R. (1999). Insulin resistance during puberty: results from clamp studies in 357 children. Diabetes, 48, 2039-2044. doi:10.2337/diabetes.48.10.2039

Morrison, J.A., Glueck, C.J., \& Wang, P. (2012). Childhood risk factors predict cardiovascular disease, impaired fasting glucose plus type 2 diabetes mellitus, and high blood pressure 26 years later at a mean age of 38 years: the Princeton-lipid research clinics follow-up study. Metabolism, 61, 531-541. doi:10.1016/j.metabol.2011.08.010

Sedgwick, M.J., Morris, J.G., Nevill, M.E., \& Barrett, L.A. (2015). Effect of repeated sprints on postprandial endothelial function and triacylglycerol concentrations in adolescent boys. Journal of Sports Sciences, 33, 806-816. doi:10.1080/02640414.2014.964749 
Senn, S. (1993). Cross-over trials in clinical research. Chichester, United Kingdom: Wiley.

Slaughter, M.H., Lohman, T.G., Boileau, R.A., Horswill, C.A., Stillman, R.J., Van Loan, M.D., \& Bemben, D.A. (1988). Skinfold equations for estimation of body fatness in children and youth. Human Biology, 60, 709-723.

Steinberger, J., Jacobs, D.R., Raatz, S., Moran, A., Hong, C.P., \& Sinaiko, A.R. (2005). Comparison of body fatness measurements by BMI and skinfolds vs dual energy X-ray absorptiometry and their relation to cardiovascular risk factors in adolescents. International Journal of Obesity, 29, 1346-1352. doi:10.1038/sj.ijo.0803026

Tanner, J.M. (1962). Growth at adolescence (2nd ed.). Oxford, United Kingdom: Blackwell Scientific Publications.

Tolfrey, K., Campbell, I.G., \& Jones, A.M. (1999). Intra-individual variation of plasma lipids and lipoproteins in prepubescent children. European Journal of Applied Physiology, 79, 449-456. doi:10.1007/s004210050536

Tolfrey, K., Engstrom, A., Murphy, C., Thackray, A., Weaver, R., \& Barrett, L.A. (2014a). Exercise energy expenditure and postprandial lipemia in girls. Medicine and Science in Sports and Exercise, 46, 239-246. doi:10.1249/MSS.0b013e3182a59ab1 Tolfrey, K., Thackray, A.E., \& Barrett, L.A. (2014b). Acute exercise and postprandial lipemia in young people. Pediatric Exercise Science, 26, 127-137. doi:10.1123/pes.2013-0126 
Wendler, D., Michel, E., Kästner, P., \& Schmahl, F.W. (1992). Menstrual cycle exhibits no effect on postprandial lipemia. Hormone and Metabolic Research, 24, 580-581. doi:10.1055/s-2007-1003394

Westerveld, H.E. (1998). Estrogens and postprandial lipid metabolism. Atherosclerosis, 141(Suppl. 1), S105-S107. doi:10.1016/S0021-9150(98)00227-5 


\section{Figure legends}

Figure 1. Diagram of the 2-day study protocol. TAG, triacylglycerol. ${ }^{+}$Evening meal replicated from first condition.

Figure 2. Fasting (F) and postprandial plasma triacylglycerol concentrations ([TAG]) in the control (CON) and high-intensity interval running (HIIR) conditions for $n=16$ girls and $n=15$ boys. Values are mean (SD). Black rectangles denote consumption of breakfast and lunch meals at 08:00 and 12:00, respectively. Main effect condition $P<$ 0.001 ; main effect sex $P=0.004$; condition-by-sex interaction $P=0.600$.

Figure 3. Individual changes (delta) in the total area under the plasma triacylglycerol (TAG) concentration versus time curve (TAUC) in the high-intensity interval running (HIIR) condition compared with the control condition (CON) in boys ( $\square$ ) and girls ( $\square$ ). A negative response indicates a reduction in TAUC-TAG in HIIR compared with CON. 
Table 1 Physical and physiological characteristics.

\begin{tabular}{lcccc}
\hline & $\begin{array}{c}\text { Boys } \\
(\boldsymbol{n}=\mathbf{1 5})\end{array}$ & $\begin{array}{c}\text { Girls } \\
(\boldsymbol{n}=\mathbf{1 6})\end{array}$ & $\begin{array}{c}\text { Girls vs. Boys } \\
\mathbf{9 5 \%} \text { CI* }\end{array}$ & Effect Size \\
\hline Age (years) & $11.8(0.4)$ & $12.1(0.7)$ & -0.7 to 0.1 & 0.49 \\
Body mass (kg) & $42.8(8.0)$ & $45.1(7.6)$ & -8.1 to 3.4 & 0.30 \\
Stature (m) & $1.53(0.09)$ & $1.55(0.09)$ & -0.09 to 0.04 & 0.28 \\
Body mass index $\left(\mathrm{kg} \cdot \mathrm{m}^{-2}\right)$ & $18.3(2.8)$ & $18.7(2.1)$ & -2.1 to 1.5 & 0.14 \\
Percent body fat $(\%)$ & $13.5(5.2)$ & $21.4(3.6)$ & -11.1 to $-4.6^{\mathrm{a}}$ & 1.75 \\
Lean body mass $(\mathrm{kg})$ & $36.7(5.4)$ & $35.4(5.5)$ & -2.7 to 5.3 & 0.24 \\
Pubic hair development** & $2(1)$ & $2(3)$ & -2 to 1 & 0.03 \\
Peak oxygen uptake $\left(\mathrm{mL} \cdot \mathrm{kg}^{-1} \cdot \mathrm{min}^{-1}\right)$ & $55(6)$ & $43(6)$ & 8 to $17^{\mathrm{a}}$ & 2.08 \\
Maximal aerobic speed $\left(\mathrm{km}^{-1} \mathrm{~h}^{-1}\right)$ & $12.5(1.6)$ & $11.5(1.1)$ & -0.04 to 1.98 & 0.70 \\
\hline
\end{tabular}

Values are mean (SD) for $n=15$ boys and $n=16$ girls. $* 95 \%$ confidence interval of the mean absolute difference between the groups. **Self-assessment - median (interquartile range).

${ }^{a}$ Significant difference between boys and girls $(P<0.001)$ 
Table 2 Energy and macronutrient intakes during the intervention day in the control (CON) and high-intensity interval running (HIIR) conditions in boys and girls.

\begin{tabular}{|c|c|c|c|c|c|c|}
\hline & \multicolumn{2}{|c|}{ Boys $(n=15)$} & \multicolumn{2}{|c|}{ Girls $(n=16)$} & \multirow{2}{*}{$\begin{array}{l}\text { Girls vs. Boys } \\
\text { 95\% CI* }\end{array}$} & \multirow{2}{*}{$\begin{array}{c}\text { CON vs. HIIR } \\
95 \% \mathrm{CI}^{*}\end{array}$} \\
\hline & CON & HIIR & CON & HIIR & & \\
\hline Energy $\left(\mathrm{MJ} \cdot\right.$ day $\left.^{-1}\right)$ & $8.0(1.7)$ & $8.1(1.7)$ & $6.5(1.4)$ & $6.4(1.4)$ & 0.6 to $2.7^{\mathrm{a}}$ & -0.4 to 0.4 \\
\hline Protein $\left(\mathrm{g} \cdot \mathrm{day}^{-1}\right)$ & $69.7(19.1)$ & $70.9(19.3)$ & $54.1(19.8)$ & $54.4(20.0)$ & 2.1 to $30.3^{\mathrm{a}}$ & -3.0 to 4.4 \\
\hline $\mathrm{CHO}\left(\mathrm{g} \cdot \mathrm{day}^{-1}\right)$ & $249(42)$ & $248(64)$ & $222(42)$ & $218(42)$ & -2 to 60 & -17 to 13 \\
\hline Fat $\left(g \cdot d^{-1} y^{-1}\right)$ & $68.4(25.8)$ & $70.5(24.6)$ & $49.1(15.8)$ & $48.5(15.4)$ & 7.3 to $35.0^{\mathrm{a}}$ & -3.7 to 5.2 \\
\hline$\%$ energy intake from protein & $14.7(2.7)$ & $15.2(3.8)$ & $13.9(3.6)$ & $14.1(3.7)$ & -1.5 to 3.4 & -0.3 to 1.0 \\
\hline \% energy intake from $\mathrm{CHO}$ & $53.7(6.6)$ & $52.0(8.6)$ & $57.9(3.9)$ & $57.6(3.7)$ & -9.1 to $-0.9^{a}$ & -2.7 to 0.7 \\
\hline$\%$ energy intake from fat & $31.6(6.3)$ & $32.8(8.3)$ & $28.2(4.6)$ & $28.3(4.6)$ & 0.02 to $8.16^{\mathrm{a}}$ & -1.0 to 2.2 \\
\hline
\end{tabular}

Values are mean (SD) for $n=15$ boys and $n=16$ girls. *95\% confidence interval of the mean absolute difference between the groups or experimental conditions.

CHO, carbohydrate.

${ }^{a}$ Significant difference between boys and girls $(P<0.05)$ 
Table 3 Average responses to the high-intensity interval running (HIIR) session.

\begin{tabular}{|c|c|c|c|c|}
\hline & $\begin{array}{c}\text { Boys } \\
(n=15)\end{array}$ & $\begin{array}{c}\text { Girls } \\
(n=16)\end{array}$ & $\begin{array}{l}\text { Girls vs. Boys } \\
\text { 95\% CI* }\end{array}$ & Effect Size \\
\hline Peak heart rate (beats $\cdot \mathrm{min}^{-1}$ )** & $200(6)$ & $203(7)$ & -9 to 1 & 0.57 \\
\hline Start interval heart rate (beats $\cdot \mathrm{min}^{-1}$ ) & $144(10)$ & $164(12)$ & -28 to $-11^{\text {a }}$ & 1.73 \\
\hline Percent peak heart rate (\%) & $72(5)$ & $80(5)$ & -12 to $-5^{a}$ & 1.65 \\
\hline End interval heart rate (beats· $\mathrm{min}^{-1}$ ) & $190(7)$ & $196(8)$ & -11.3 to $-0.1^{\mathrm{a}}$ & 0.75 \\
\hline Percent peak heart rate (\%) & $95(3)$ & $96(2)$ & -3 to 1 & 0.43 \\
\hline Rating of perceived exertion & $16(1)$ & $14(2)$ & 0.2 to $2.6^{\mathrm{a}}$ & 0.85 \\
\hline
\end{tabular}

Values are mean (SD) for $n=15$ boys and $n=16$ girls. *95\% confidence interval of the mean absolute difference between the groups. **Peak heart rate was determined during the speedbased incremental treadmill protocol.

a Significant difference between boys and girls $(P<0.05)$ 
Table 4 Fasting and postprandial plasma triacylglycerol and glucose concentrations in the control (CON) and high-intensity interval running (HIIR) conditions in boys and girls.

\begin{tabular}{|c|c|c|c|c|c|c|}
\hline & \multicolumn{2}{|c|}{ Boys $(n=15)$} & \multicolumn{2}{|c|}{ Girls $(n=16)$} & \multirow{2}{*}{$\begin{array}{c}\text { Girls vs. Boys } \\
\text { 95\% CI* }\end{array}$} & \multirow{2}{*}{$\begin{array}{c}\text { CON vs. HIIR } \\
95 \% \text { CI* }^{*}\end{array}$} \\
\hline & CON & HIIR & $\mathrm{CON}$ & HIIR & & \\
\hline \multicolumn{7}{|l|}{ Triacylglycerol } \\
\hline Fasting $\left(\mathrm{mmol} \cdot \mathrm{L}^{-1}\right)$ & $0.56(0.49$ to 0.65$)$ & $0.52(0.45$ to 0.60$)$ & $0.87(0.75$ to 1.00$)$ & $0.74(0.64$ to 0.85$)$ & -44 to $-18 \%{ }^{a}$ & -18 to $-5 \%^{\mathrm{b}}$ \\
\hline TAUC $\left(\mathrm{mmol} \cdot \mathrm{L}^{-1} 6.5 \mathrm{~h}\right)$ & $6.35(5.44$ to 7.41$)$ & $5.67(4.85$ to 6.61$)$ & 8.63(7.43 to 10.02$)$ & $7.77(6.69$ to 9.02$)$ & -40 to $-10 \%{ }^{a}$ & -16 to $-5 \%{ }^{b}$ \\
\hline iAUC $\left(\mathrm{mmol} \cdot \mathrm{L}^{-1} 6.5 \mathrm{~h}\right)$ & $2.54(1.76$ to 3.64$)$ & $2.20(1.53$ to 3.15$)$ & $2.21(1.56$ to 3.15$)$ & $2.82(1.98$ to 4.00$)$ & -38 to $44 \%$ & -21 to $40 \%$ \\
\hline \multicolumn{7}{|l|}{ Glucose } \\
\hline Fasting $\left(\mathrm{mmol} \cdot \mathrm{L}^{-1}\right)$ & $5.51(5.28$ to 5.75$)$ & $5.42(5.20$ to 5.65$)$ & $5.71(5.48$ to 5.95$)$ & $5.67(5.45$ to 5.91$)$ & -9 to $1 \%$ & -3 to $1 \%$ \\
\hline TAUC $\left(\mathrm{mmol} \cdot \mathrm{L}^{-1} 6.5 \mathrm{~h}\right)$ & $42.5(41.2$ to 43.9$)$ & $42.3(40.9$ to 43.6$)$ & $42.4(41.1$ to 43.7$)$ & $43.8(42.5$ to 45.2$)$ & -6 to $2 \%$ & -1 to $4 \%$ \\
\hline $\mathrm{iAUC}\left(\mathrm{mmol} \cdot \mathrm{L}^{-1} 6.5 \mathrm{~h}\right)$ & $6.59(4.92$ to 8.84$)$ & $6.50(4.85$ to 8.71$)$ & $4.73(3.56$ to 6.28$)$ & $6.43(4.84$ to 8.54$)$ & -14 to $63 \%$ & -11 to $51 \%$ \\
\hline
\end{tabular}

Values are geometric mean (95\% confidence interval) for $n=15$ boys and $n=16$ girls. Statistical analyses are based on natural log transformed data. $* 95 \%$ confidence interval for the ratio of geometric means.

TAUC, total area under the concentration versus time curve; iAUC, incremental area under the concentration versus time curve.

a Significant difference between boys and girls $(P<0.05)$

${ }^{\mathrm{b}}$ Significant difference between HIIR and CON $(P<0.05)$ 
Figure 1

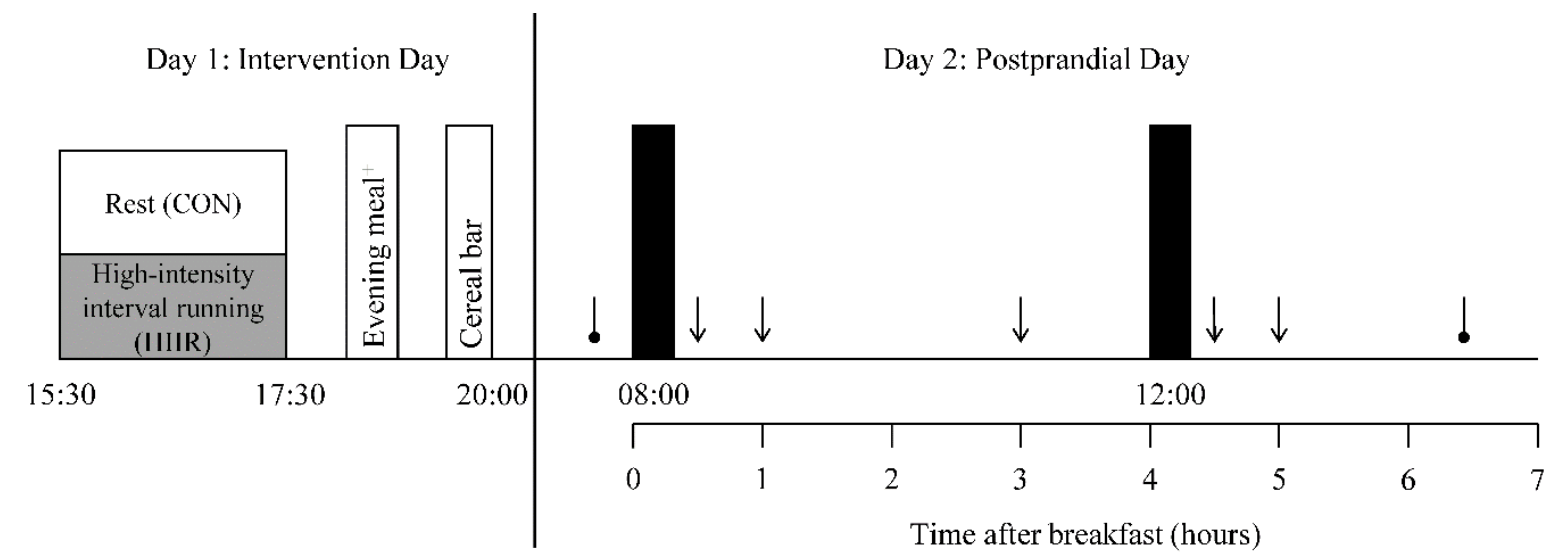

Key:

Test meals
$\downarrow$ Capillary blood sample for [TAG] and [glucose]

- Capillary blood sample for [TAG], [glucose], [haemoglobin] and haematocrit 
Figure 2

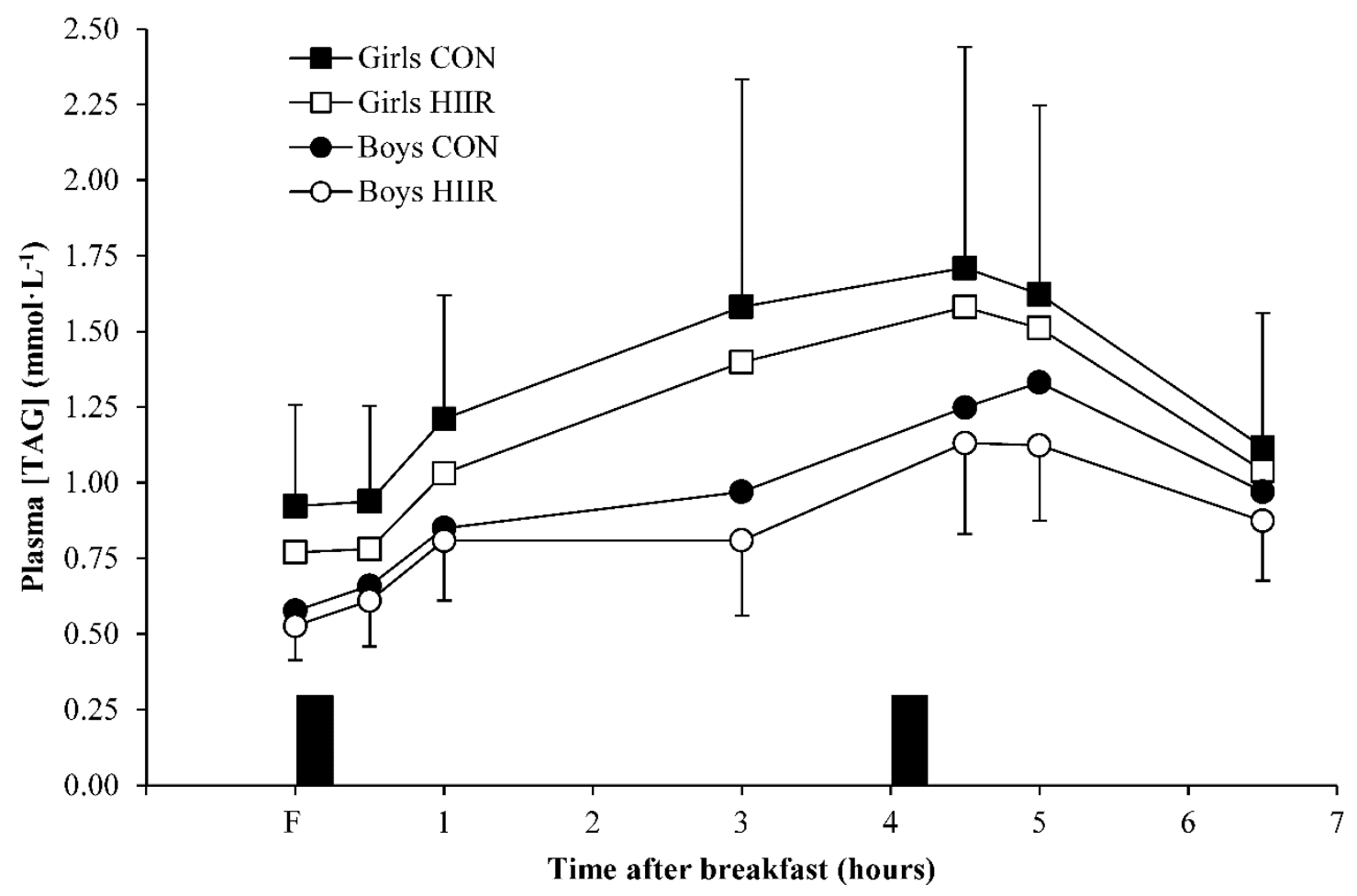


Figure 3

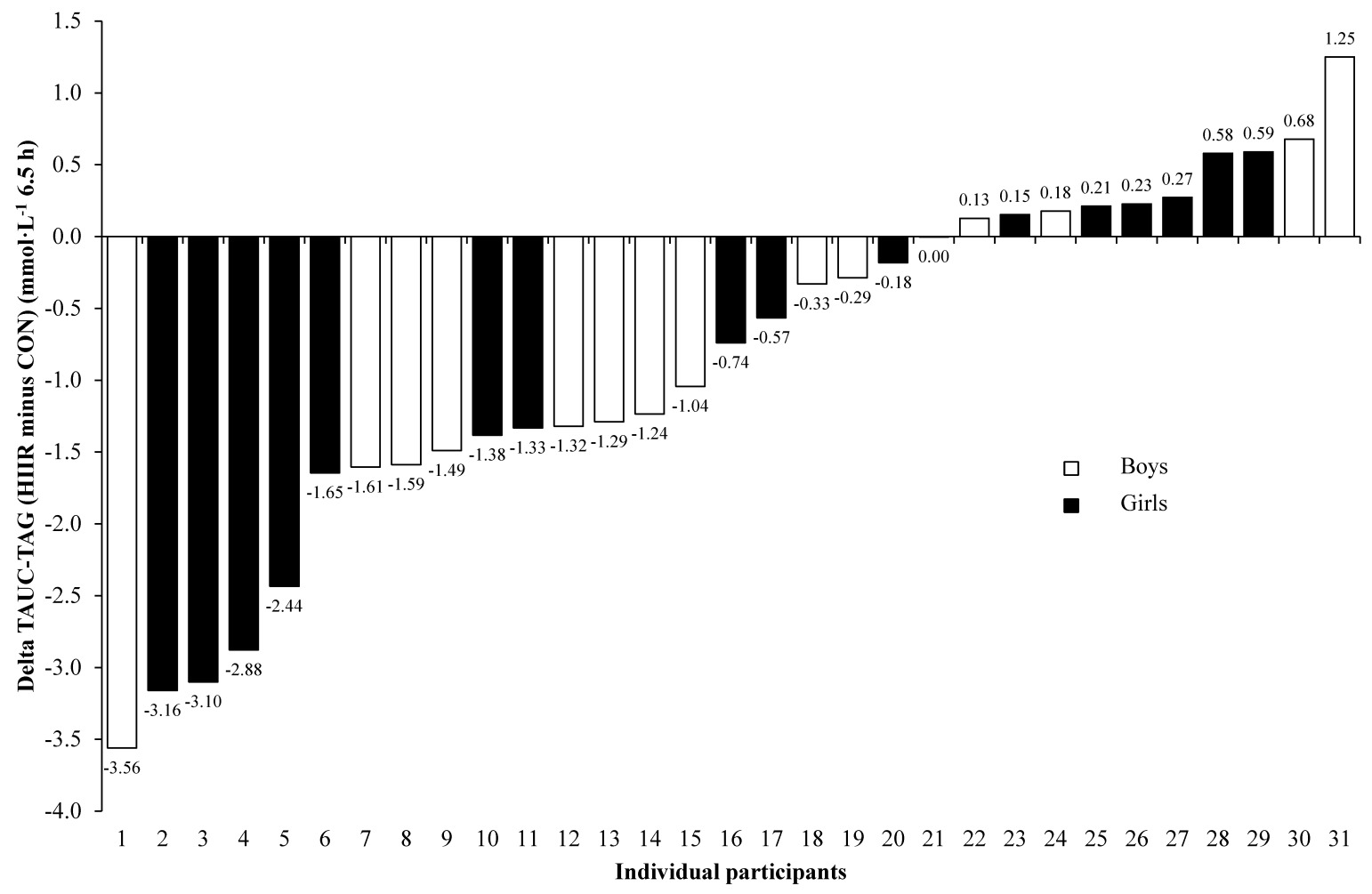

